\title{
Difference in the heliospheric modulation of cosmic-ray protons and electrons during the solar minimum period of 2006 to 2009
}

\author{
M. S. Potgieter and E. E. Vos
}

Center for Space Research, North-West University, 2520 Potchefstroom, South Africa
e-mail: marius. potgieter@nwu.ac.za

Received 2 November 2016 / Accepted 8 January 2017

\begin{abstract}
Context. The PAMELA experiment observed galactic proton and electron spectra down to $70 \mathrm{MV}$ and $400 \mathrm{MV}$, respectively, from mid-2006 to the end of 2009 during what is called an $A<0$ solar magnetic polarity cycle. During this period, solar activity was at its lowest level since the beginning of the space exploration era. This provides the opportunity to study charge-sign-dependent modulation under very quiet heliospheric conditions.

Aims. Drift theory for the solar modulation of cosmic rays predicts that the intensity of protons at the Earth is expected to show a different rate of recovery towards solar minimum when compared to electrons during $A<0$ cycles. These charge-sign related differences are investigated.

Methods. The solutions of a comprehensive three-dimensional drift model are compared to PAMELA spectra to authenticate the modelling approach and then to make predictions of how electrons and protons are differently modulated down to $1 \mathrm{MeV}$, based on new very local interstellar spectra.

Results. The comparison of observations and modelling provides insight into how the rigidity dependence of the three major diffusion coefficients changes during such quiet modulation conditions. How drift effects dissipate above several $\mathrm{GeV}$ and below $100 \mathrm{MeV}$ is illustrated for both protons and electrons. The modulation that occurred at the Earth during this quiet period is shown as a function of rigidity and time. The $\mathrm{e}^{-} / \mathrm{p}$ ratio is computed from $10 \mathrm{MV}$ to $50 \mathrm{GV}$ for this period and a prediction is made for what may be observed in terms of spectra during the next $A>0$ solar minimum.

Conclusions. The presence of drifts during this quiet period is established based on the presented modelling and PAMELA measurements. Drift effects for protons and electrons are quantified in terms of their rigidity and temporal development from 2006 to 2009.
\end{abstract}

Key words. Sun: heliosphere - solar-terrestrial relations - Sun: activity

\section{Introduction}

According to drift theory for cosmic rays (CRs) in the heliosphere, the intensity of positively charged CRs at Earth is expected to show a different rate of recovery towards solar minimum modulation conditions when compared to negatively charged CRs during so-called $A<0$ magnetic polarity cycles. During these cycles, the heliospheric magnetic field (HMF) is directed outward in the northern and inward in the southern hemisphere, causing protons to drift from the heliospheric boundary inwards towards the Earth mainly through the equatorial regions of the heliosphere, while electrons drift inwards mainly through the polar regions of the heliosphere. Protons then encounter the changing heliospheric current sheet (HCS) so that their recovery to solar minimum modulation depends to a large extent on the decreasing rate of the tilt angle of the HCS (Ferreira \& Potgieter 2003). For elaborate illustrations of how CR particles actually drift and propagate in the heliosphere, we refer to Strauss et al. (2012a).

Because of this drift behaviour of charged particles, the intensity-time profile of $\mathrm{CR}$ protons before and after solar minimum modulation forms a peak during $A<0$ magnetic polarity cycles, whereas electrons, being less sensitive to what happens to the HCS tilt angle, typically exhibit an intensity-time profile that is less peaked. If CR drifts would dominate the modulation process, the profile for protons would be sharply peaked while the profile for electrons would be flattish. Of course, when the
HMF changes its polarity (to an $A>0$ cycle), protons will exhibit the flatter profile while electrons exhibit the sharper profile. This constitutes what is known as charge-sign-dependent modulation caused by $\mathrm{CR}$ drifts.

This 22-yr cyclic behaviour of CR protons, related to the polarity of the HMF and the waviness of HCS, was first predicted and illustrated with a basic numerical model by Jokipii \& Thomas (1981) and later comprehensively described by Kóta \& Jokipii (1983) and Potgieter \& Moraal (1985); see also le Roux \& Potgieter (1995). This predicted 22-yr pattern has been observed with CR detectors on ground level (called neutron monitors) since the late 1950s; see also the reviews by Strauss et al. (2012b) and Potgieter (2013).

The research presented here is focused on the abovementioned charge-sign-dependent behaviour of CRs in the heliosphere. The specific difference in the behaviour of CR protons and electrons, caused by particle drift, was first illustrated by Potgieter \& Moraal (1985). They reported that when the CR intensity ratio of the 1965 ( $A<0$ epoch) to the 1977 ( $A>0$ epoch) solar minimum periods were plotted as a function of kinetic energy, the protons behaved quite differently from the electrons, as predicted by their drift modulation model. The differences in the solar modulation of CR protons, electrons and helium as a function of the tilt angle of the HCS were comprehensively modelled (Potgieter \& Burger 1990), with later models even including the effects of the solar wind termination shock (Langner \& Potgieter 2004a) on electron-positron (Potgieter \& Langner 2004) and 
proton-antiproton modulation (Langner \& Potgieter 2004b). In particular, it was predicted how the electron to proton (or helium) ratio would change as a function of HCS tilt angle over a complete 22-yr cycle (Potgieter et al. 2001). These predictions could eventually be compared to long-term observations (as opposed to short duration balloon flights) when the Ulysses mission became operational and reported observations of electrons (at 1.2 and $2.5 \mathrm{GV}$ ), protons (at 2.5 GV) and helium (at $1.2 \mathrm{GV}$ ), specifically for the $A>0$ period from late 1990 to 2000, with a polarity change in 2000-2001 (Heber et al. 2002, 2009). It was found that the helium and proton intensity-time profiles were indeed broader and flatter than the corresponding electron profile.

However, a direct comparison between these Ulysses observations and contemporary drift models indicated that the drift effects on CR modulation were overestimated, particularly around solar maximum activity. For example, the electron to proton (or helium) ratio for an $A>0$ cycle was predicted to have a prominent "W-shape" in $A>0$ cycles, when shifting from maximum to minimum and back to maximum solar activity, while the observations exhibited a rather flat profile, more like a flat, small "w". At a later stage, the latter was extensively modelled by Ndiitwani et al. (2005) who used a time-dependent drift model. These efforts triggered theoretical studies of drift theory, which is still ongoing (see e.g. Engelbrecht \& Burger 2015 and Ngobeni \& Potgieter 2015). Evidently, the role of CR drifts in causing charge-sign-dependent modulation over a complete 22-yr cycle is not yet fully understood.

The Payload for Antimatter Matter Exploration and Lightnuclei Astrophysics (PAMELA) space bound experiment (Picozza et al. 2007; Menn et al. 2013; Adriani et al. 2014) provided a new opportunity to study charge-sign-dependent modulation, this time for the $A<0$ cycle from June 2006, when the mission started, to the end of 2009. During this period, PAMELA observed differential fluxes down to $400 \mathrm{MV}$ for protons (Adriani et al. 2013), and down to $80 \mathrm{MV}$ for electrons (Adriani et al. 2015). This long-duration mission allowed for a continuous collection of data during this period and the good statistics made it possible to average the proton flux over each Carrington rotation period; but because of lower statistics, the electron fluxes were averaged over consecutive six-month periods.

The PAMELA data prompted Potgieter et al. (2014, 2015) and Vos \& Potgieter (2015) to study the modulation of galactic protons and electrons using a comprehensive three-dimensional (3D) modulation model that includes drifts and all the other important modulation processes. They focussed on measurements of CR protons and electrons between July 2006 and December 2009 (Adriani et al. 2013, 2015) as is discussed and illustrated below. See also the complimentary modelling by Zhao et al. (2014).

It is, by now, well-known that heliospheric modulation conditions were exceptionally quiet during the mentioned time period. This was discussed in detail by Potgieter et al. (2014), who gave several references to other independently made observations. The availability of precision measurements of protons and electrons from PAMELA for this period can be considered as very fortunate since it made possible a detailed investigation of CR drifts under these optimal solar minimum conditions.

From a global modulation point of view, another fortunate event took place in August 2012, when Voyager 1 became the first spacecraft to cross the heliopause (HP). For the first time, insitu observations for both protons and electrons have been made below $100 \mathrm{MeV}$ beyond the HP. This allows for the construction of far more precise very local interstellar spectra (VLISs) at these low energies, as discussed in length by Potgieter (2014b). These new VLISs are given in the next section as a requisite for doing the global modelling of these CRs properly for 2006 to 2009.

The aim of this study is to build on the modelling of Potgieter et al. (2015) and Vos \& Potgieter (2015) who computed proton and electron spectra for the period and energy range mentioned above. These previous modelling efforts are considered as a validation of the $3 \mathrm{D}$ model for this extraordinarily quiet modulation period. The particular set of modulation parameters established for this purpose was also used by Vos \& Potgieter (2016) for a study on the global gradients of galactic protons using PAMELA and Ulysses/KET (Kiel Electron Telescope) measurements (Gieseler \& Heber 2016). For the current study, this parameter set is briefly discussed and then applied but with the focus on modelling the differences between CR protons and electrons down to very low energies for the mentioned period. By emphasising these differences, the way in which drift effects dissipate particularly below $100 \mathrm{MeV}$, and also above several $\mathrm{GeV}$, is studied and illustrated for both protons and electrons.

First, the respective new VLIS for electrons and protons is shown, followed by a short discussion of the numerical model and the mentioned set of parameters, including the diffusion and drift coefficients. The differences between electron and proton modulation are then illustrated in terms of their respective rigidity (and kinetic energy) spectra, with emphasis on the effects of drifts related to the two HMF polarity cycles and over a rigidity range from $10 \mathrm{MV}$ to $30 \mathrm{GV}$. The predicted electron to proton ratio for the period 2006 to 2009 down to $10 \mathrm{MV}$ is shown and discussed, ending with a summary and conclusions.

\section{Numerical simulation of PAMELA spectra}

\subsection{Local interstellar spectra}

Computational modelling of CR modulation as mentioned above requires that a VLIS has to be specified as model input (handled as an initial condition) for protons and electrons, respectively, at the boundary of the simulated heliosphere, assumed to be the HP. Until recently, neither the absolute flux nor the spectral shape of these VLISs could be determined with adequate precision across the entire energy range where solar modulation plays an important role. This situation improved significantly when Voyager 1 became the first spacecraft to cross the HP at a heliocentric distance of 122 AU. Measurements of CR electrons and protons taken from beyond the HP (Stone et al. 2013 and Webber \& McDonald 2013) are assumed to reflect CR intensity levels for the very local interstellar medium. Vos \& Potgieter (2015) used these measurements as benchmark intensities for constructing a proton VLIS below $\sim 600 \mathrm{MeV}$. Above $\sim 30 \mathrm{GeV}$, where solar modulation has little to no effect on galactic CRs (see Strauss \& Potgieter 2014a), PAMELA and AMS-02 proton observations were used as VLIS-equivalent intensity levels (Adriani et al. 2013; Aguilar et al. 2013). By combining these measurements, and using applications of the GALPROP model (Strong et al. 2011) as a guide for interpolating between $600 \mathrm{MeV}$ and $30 \mathrm{GeV}$, a new proton VLIS is constructed and utilised for this modelling study. A similar approach was followed for the electron VLIS, this time using Voyager 1 (Stone et al. 2013), PAMELA (Adriani et al. 2015) and AMS02 (Aguilar et al. 2014) electron observations. We also refer to Bisschoff \& Potgieter (2014) who used the GALPROP model to investigate the implications of these Voyager 1 electron measurements on galactic propagation. 


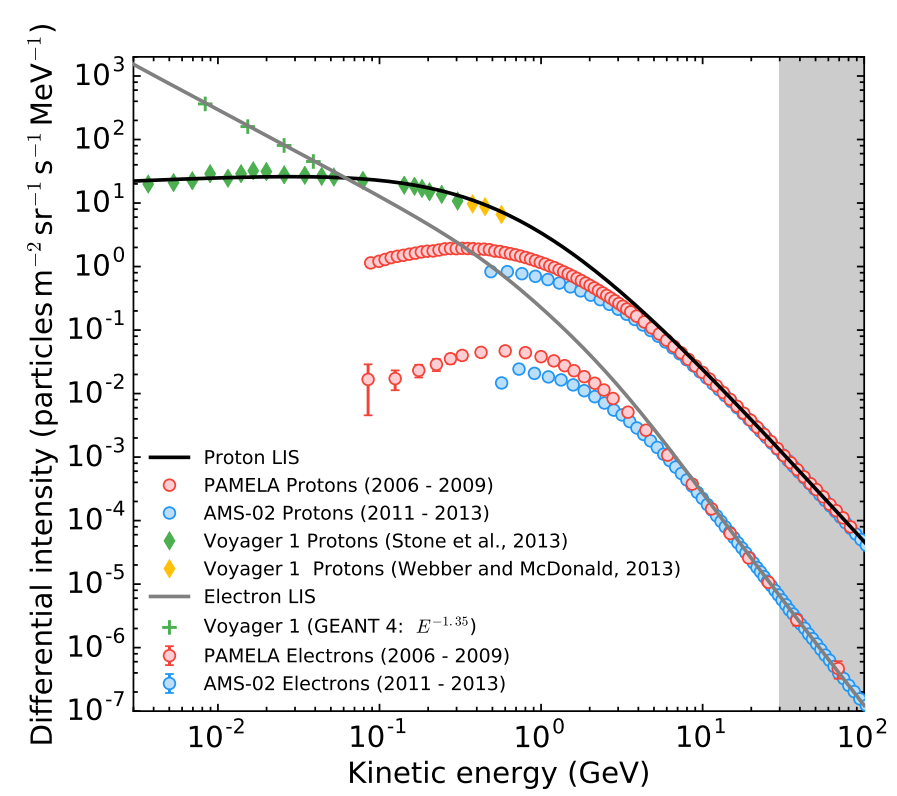

Fig. 1. A very local interstellar spectrum for galactic protons (black line) and for galactic electrons (grey line), specified in the model as unmodulated input spectra at the boundary $(122 \mathrm{AU})$ of the simulated heliosphere. These spectra were constructed by combining PAMELA (red circles) and AMS-02 (blue circles) measurements at the Earth above $30 \mathrm{GeV}$ (grey shaded band), and Voyager 1 measurements below $\sim 600 \mathrm{MeV}$ taken from beyond the HP (diamonds and crosses). References to the observations are given in the text.

Figure 1 shows the mentioned Voyager 1, PAMELA and AMS-02 observations overlaid by the newly constructed proton VLIS (black line) and electron VLIS (grey line). The shaded band above $30 \mathrm{GeV}$ represents the region where modulation effects can be neglected and where these VLIS are normalised to the measurements. The Voyager 1 electron measurements below $\sim 40 \mathrm{MeV}$ are based on response functions derived from GEANT 4 simulations (green crosses), indicating an $E^{-1.35}$ spectral index that is used for this work (see also the discussions by Stone et al. 2013; Bisschoff \& Potgieter 2014; Potgieter et al. 2015; Nndanganeni \& Potgieter 2016; Corti et al. 2016).

Mathematical expressions for the VLIS for protons and electrons are given, respectively, by

$J_{p}=2.70 \frac{E^{1.12}}{\beta^{2}}\left(\frac{E+0.67}{1.67}\right)^{-3.93}$,

and

$J_{e^{-}}=\frac{0.21 E^{-1.35}}{\beta^{2}}\left(\frac{E^{1.65}+0.6920}{1.6920}\right)^{-1.1515}+J_{\text {bump }}$,

with

$J_{\text {bump }}=1.73 \exp \left(4.19-5.40 \log E-8.9 E^{-0.64}\right)$,

where $E$ is the kinetic energy in $\mathrm{GeV}, \beta$ the ratio of $\mathrm{CR}$ particle speed relative to the speed of light, and $j=P^{2} f$ is the differential intensity in units of particles $\mathrm{m}^{-2} \mathrm{sr}^{-1} \mathrm{~s}^{-1} \mathrm{MeV}^{-1}$, with $f(\boldsymbol{r}, P, t)$, the galactic CR distribution function at a vector position $\boldsymbol{r}$ (specified in 3D by heliocentric coordinates $r, \theta$ and $\phi$ ) for a given rigidity $P$ (in $\mathrm{GV}$ ) and time $t$. The incorporation of the "bump" in the VLIS for electrons between 5 and $15 \mathrm{GeV}$, which is hardly visible in Fig. 1, was discussed at length by Potgieter (2014b) and Potgieter et al. (2015). It does not change the conclusions of the work reported here, but it does affect the values of the diffusion coefficients that are needed for electrons in the model to reproduce the observed spectra in this particular energy range.

\subsection{Numerical modulation model}

A full 3D modulation model is used to compute modulated differential intensities throughout the heliosphere. It is based on the numerical solution of the heliospheric transport equation from Parker (1965):

$\frac{\partial f}{\partial t}=-\left(\boldsymbol{V}+\left\langle\boldsymbol{v}_{A}\right\rangle\right) \cdot \nabla f+\nabla \cdot\left(\boldsymbol{K}_{s} \cdot \nabla f\right)+\frac{1}{3}(\nabla \cdot \boldsymbol{V}) \frac{\partial f}{\partial \ln P}$,

with $f, t$ and $P$ as before. For calculating steady-state solutions, as was done for this study, the term $\partial f / \partial t$ is set to zero, which is a reasonable assumption for modulation during solar minimum when modulation parameters change gradually. The terms on the right-hand side respectively represent convection, with $\boldsymbol{V}$ the solar wind (SW) velocity, averaged particle drift velocity $\left\langle\boldsymbol{v}_{A}\right\rangle$ caused by gradients, curvatures, and HCS drifts in the global HMF, diffusion, with $\boldsymbol{K}_{S}$ the symmetric diffusion tensor, and adiabatic cooling. For solar minimum activity, the magnitude of $\boldsymbol{V}$ is assumed to have a strong latitudinal dependence as described in detail by Potgieter et al. (2014); we refer to their Equation 12, which also contains the decrease of $\boldsymbol{V}$ across the termination shock into the heliosheath. The acceleration effects of the termination shock as well as any energy gain process inside the heliosheath are neglected for the galactic CRs considered here; for discussions of such possible effects, we refer to Langner et al. (2006), Giacalone et al. (2012), le Roux et al. (2016) and Prinsloo et al. (2017), for example. However, the modulation effect of the heliosheath has been incorporated consistently through the expressions for the diffusion coefficients. The specifics of how this is done are described in detail by Potgieter et al. (2014, 2015); an explanation of how representative values for the HCS tilt angle $(\alpha)$ and the HMF at the Earth $\left(B_{e}\right)$ are calculated for the period 2006 to 2009 is also provided. Illustrations of the global latitudinal and radial proton intensity gradients, for the latter from the Earth up to the heliopause, based on these assumptions and the model presented here, are shown and discussed by Vos \& Potgieter (2016). Cosmic ray modulation beyond the heliopause is not considered; for the details of such an approach, we refer to, for example, Luo et al. (2016) and references therein.

\subsection{Diffusion and drift coefficients}

Figure 2 shows the rigidity dependence of the parallel $\left(\lambda_{\|}\right)$and perpendicular $\left(\lambda_{\perp}\right)$ mean-free paths (MFPs), along with the drift scale $\left(\lambda_{A}\right)$, for protons (top panel) and electrons (bottom panel), from the second half of 2006 (indicated as 2006b) to the second half of 2009 (indicated as 2009b). Similar to Potgieter et al. (2014, 2015), it was found that the changes in particle MFPs and the drift scale shown in the figure were required to reproduce proton and electron spectra measured by PAMELA in detail, as shown and discussed later in this article. These changes include the combined contribution of the yearly weakening average HMF magnitude and the temporal development of $\alpha$, as well as additional MFP increases that changes in $\alpha$ and $B_{e}$ could not account for.

The diffusion coefficients (DCs; $\kappa$ ) are related to the particle MFPs $(\lambda)$ through $\kappa=\lambda(v / 3)$, with $v$, the particle speed. A general expression for the MFP parallel to the average background 


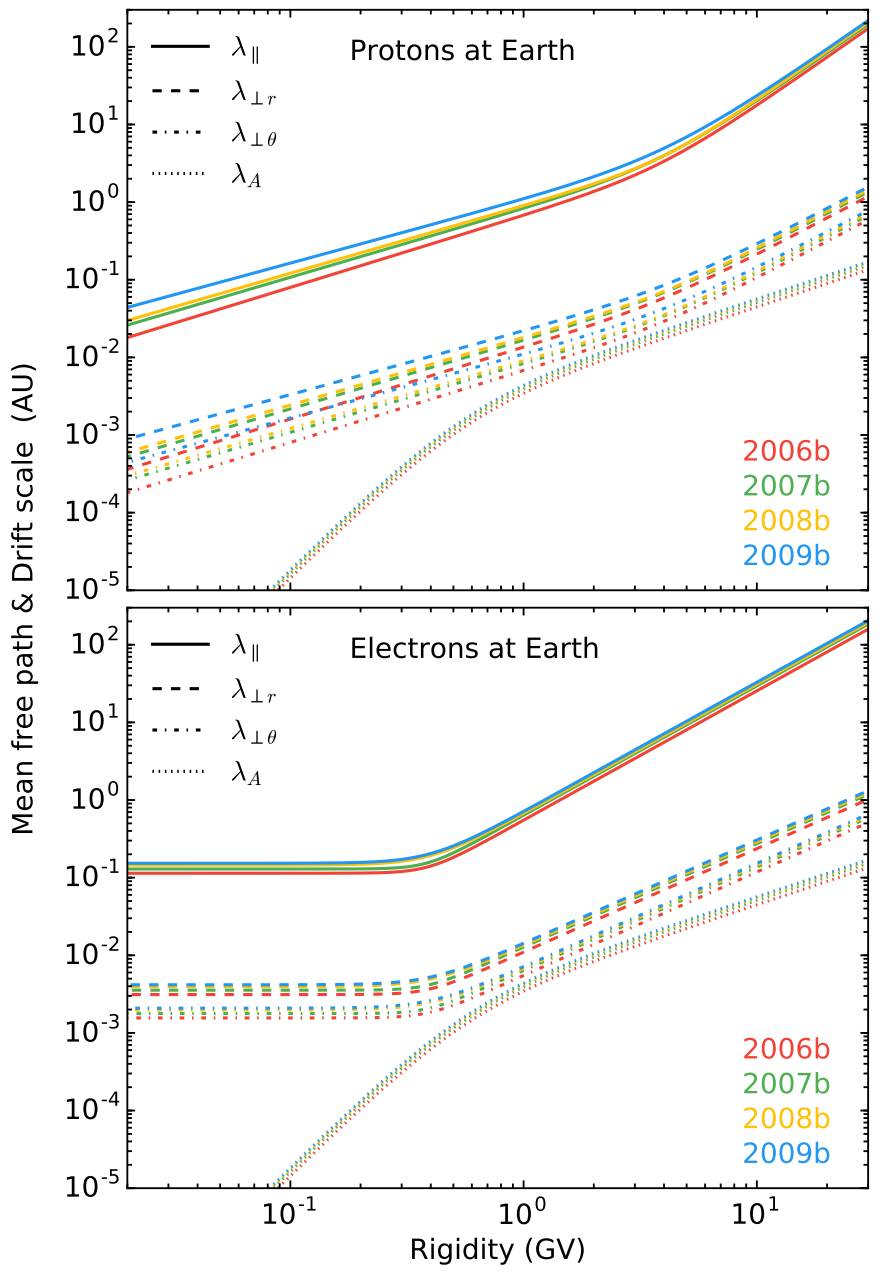

Fig. 2. Proton (top) and electron (bottom) MFPs are shown, as required for reproducing CR spectra measured by PAMELA between July 2006 and December 2009. The solid, dashed and dashed-dotted lines represent the parallel MFPs $\left(\lambda_{\|}\right)$, and the perpendicular MFPs in the radial $\left(\lambda_{\perp r}\right)$ and polar $\left(\lambda_{\perp \theta}\right)$ directions, respectively. The lowest dotted lines represent the drift scale $\left(\lambda_{A}\right)$. As indicated by the legend, the modulation parameters from the second half of 2006 (2006b) to the second half of 2009 (2009b) are given by the coloured lines changing from red to blue.

\section{$\mathrm{HMF}$ is given by}

$\kappa_{\|}=\kappa_{\| 0} \beta F(r, \theta, \phi) G(P)$,

with $\kappa_{\| 0}$ a scaling constant in units of $\mathrm{cm}^{2} \mathrm{~s}^{-1}, r$ the radial distance, $\theta$ the polar angle, and $\phi$ the azimuthal angle, where

$F(r, \theta, \phi)=\frac{B_{0}}{B}$

and

$G(P)=\left(\frac{P}{P_{0}}\right)^{a}\left\{\frac{\left(\frac{P}{P_{0}}\right)^{c}+\left(\frac{P_{k}}{P_{0}}\right)^{c}}{1+\left(\frac{P_{k}}{P_{0}}\right)^{c}}\right\}^{\frac{b-a}{c}}$.

In Eq. (6), $B$ is the HMF magnitude in nT, with $B_{0}$ a constant that normalizes $B$ to a value of $B_{e}$ at Earth and $\beta$ the ratio of particle speed to the speed of light. The variables $a, b, c$ and $P_{k}$ determine the shape of the combined power-laws for the MFP rigidity dependence, which is different for protons and electrons, as was discussed in detail by Potgieter et al. (2014, 2015).
The slope of the rigidity dependence for the proton MFPs below $\sim 3 \mathrm{GV}$ changed from $P^{0.93}$ at the end of 2006 to $P^{0.82}$ at the end of 2009 . Above $\sim 3 \mathrm{GV}$, the slope gradually steepens to a $P^{2.1}$ dependence. For electrons, the MFPs remain independent of rigidity below $0.3 \mathrm{GV}$ before steepening to a $P^{1.67}$ slope towards higher rigidities. The perpendicular MFPs in the radial and polar directions are respectively scaled by $2 \%$ and $1 \%$ of $\lambda_{\|}$. Expressions for these coefficients are given by:

$\kappa_{\perp r}=0.02 \kappa_{\| 0} \beta F(r, \theta, \phi) G(P)$,

and

$\kappa_{\perp \theta}=0.01 \kappa_{\| 0} \beta F(r, \theta, \phi) G(P) H_{\perp \theta}$,

with $F$ and $G$ defined as before. In the above equation, $H_{\perp \theta}$ is given by

$H_{\perp \theta}=A^{+} \mp A^{-} \tanh \left[8\left(\theta_{A}-90^{\circ} \pm \theta_{F}\right)\right]$,

with $A^{ \pm}=(3 \pm 1) / 2, \theta_{F}=35^{\circ}, \theta_{A}=\theta$ for $\theta \leq 90^{\circ}$ but $\theta_{A}=180^{\circ}-\theta$ for $\theta>90^{\circ}$. The net effect of Eq. (10) is that it enhances $\kappa_{\perp}$ towards the poles, as applied by Potgieter (2000) and Ngobeni \& Potgieter (2015) and references therein.

An expression for the drift coefficient is given by

$\kappa_{A}=\frac{\beta P}{3 B} \frac{\left(\frac{P}{P_{A 0}}\right)^{2}}{1+\left(\frac{P}{P_{A 0}}\right)^{2}}$,

where drifts are reduced below $P_{A 0}=0.55 \mathrm{GV}$ relative to the weak scattering case which states that drifts scale proportionally to $\beta P$ (e.g. Ngobeni \& Potgieter 2015). This reduction in drifts is required to explain the small latitudinal gradients observed by Ulysses (Heber et al. 1997; Burger et al. 2000; Heber \& Potgieter 2006). It suffices to say that the magnitude of the HMF is modified in the polar regions of the heliosphere according to Smith \& Bieber (1991), following Raath et al. (2016). It was shown by Potgieter et al. (2014) to what extent gradient, curvature and current sheet drifts contributed to the high CR intensities observed by PAMELA during 2009, and is not repeated here.

The largely phenomenological approach followed in this study for establishing the values of the diffusion coefficients, approximates quasi-linear theory (Potgieter 1996, 2000; and reviewed by Shalchi 2009) and also adheres to constraints that follow from recent independent modulation studies (e.g. Luo et al. 2013; Zhao et al. 2014). The basic rigidity dependence of the diffusion coefficients for electrons at lower rigidities as reported here, was predicted by Teufel \& Schlickeiser (2003). For an alternative approach to electron modulation based on a two-component turbulence transport model, we refer to Engelbrecht \& Burger (2013).

It is inferred from Fig. 2 that as the heliosphere approached solar minimum conditions toward the end of 2009, the HMF became more organised (e.g. McComas et al. 2008; Aslam \& Badruddin 2012; Chowdhury et al. 2016), which may have lead to a reduction in turbulence levels and, in turn, an increase from year to year of both particle MFPs and drift scale.

\subsection{Computed spectra}

Comparing the modulation of protons and electrons consistently, the observational time periods and respective rigidities for these CRs need to coincide. Consequently, multiple PAMELA 


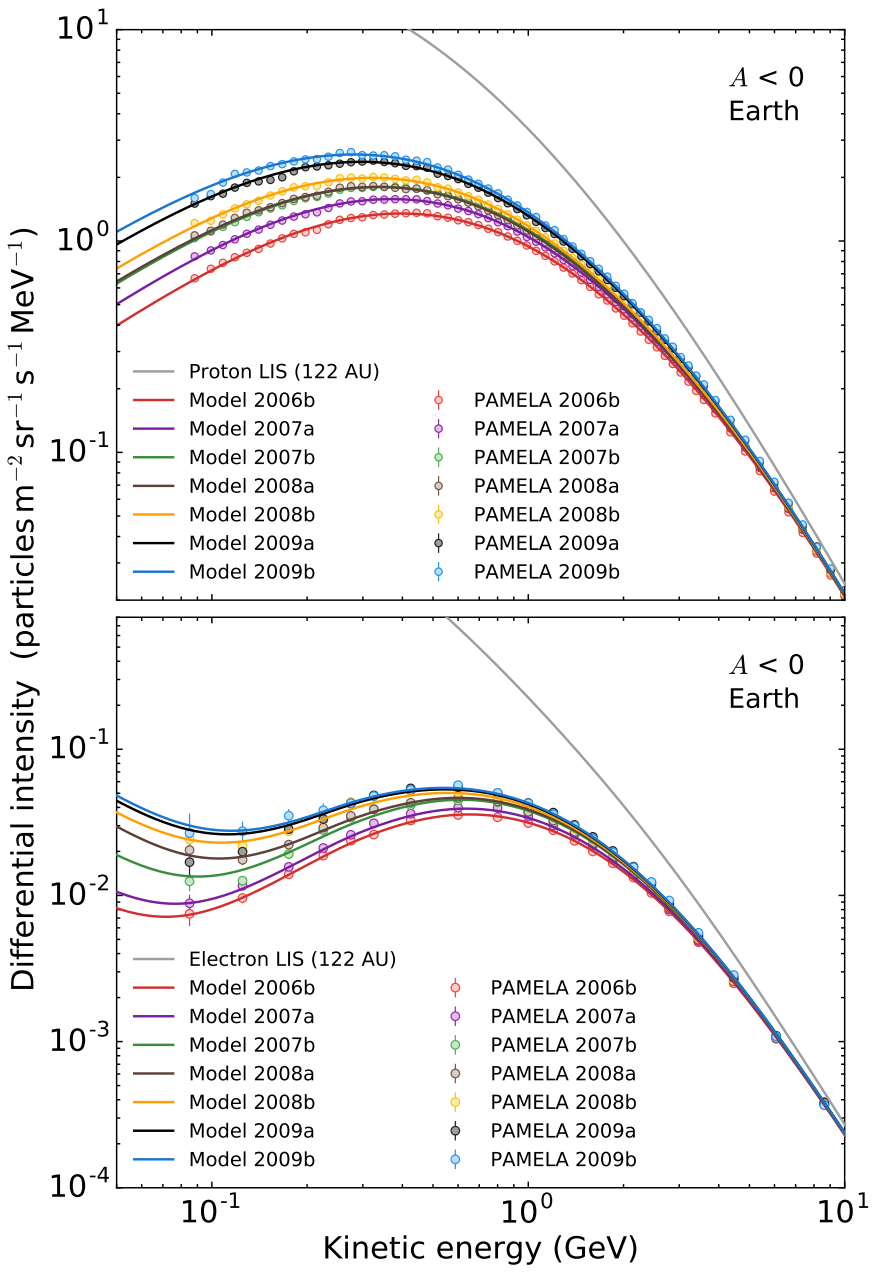

Fig. 3. PAMELA energy spectra for galactic CR protons (top) and electrons (bottom) are given by the coloured circles, and are overlaid by modelled spectra (solid lines) of corresponding colour. They are computed for an $A<0$ HMF polarity configuration, with respect to the VLIS specified at $122 \mathrm{AU}$ (grey lines). PAMELA measurements were reported by Adriani et al. $(2013,2015)$.

Carrington rotation spectra from Adriani et al. (2013) were combined to calculate averaged proton spectra at 6-month intervals, similar to the PAMELA electron spectra from Adriani et al. (2015). Previously, Potgieter et al. (2014) presented solutions that reproduced a selection of intermittent Carrington rotation proton spectra over the same period.

After incorporating the necessary changes in $\alpha$ and $B_{e}$ into the modulation model, the DCs were refined to reproduce a selection of seven half-yearly averaged PAMELA proton and electron spectra, which are given by the coloured symbols in Fig. 3, overlaid by model solutions (coloured lines). These half-year spectra are from here-on referred to as the 2006b, 2007a, 2007b, 2008a, 2008b, 2009a and 2009b spectra, where the suffixes "a" and " $b$ " indicate the first and second half-year periods, respectively. It is evident that the model correctly reproduces the temporal development of the proton (top panel) and electron (bottom panel) energy spectra observed by PAMELA, and serves as vindication of the 3D approach in this work.

The PAMELA measurements indicate that larger increases in intensity occurred at lower energies, where CRs are more responsive to changes in modulation conditions. The spectra from 2006 to 2009 clearly became progressively softer as more and

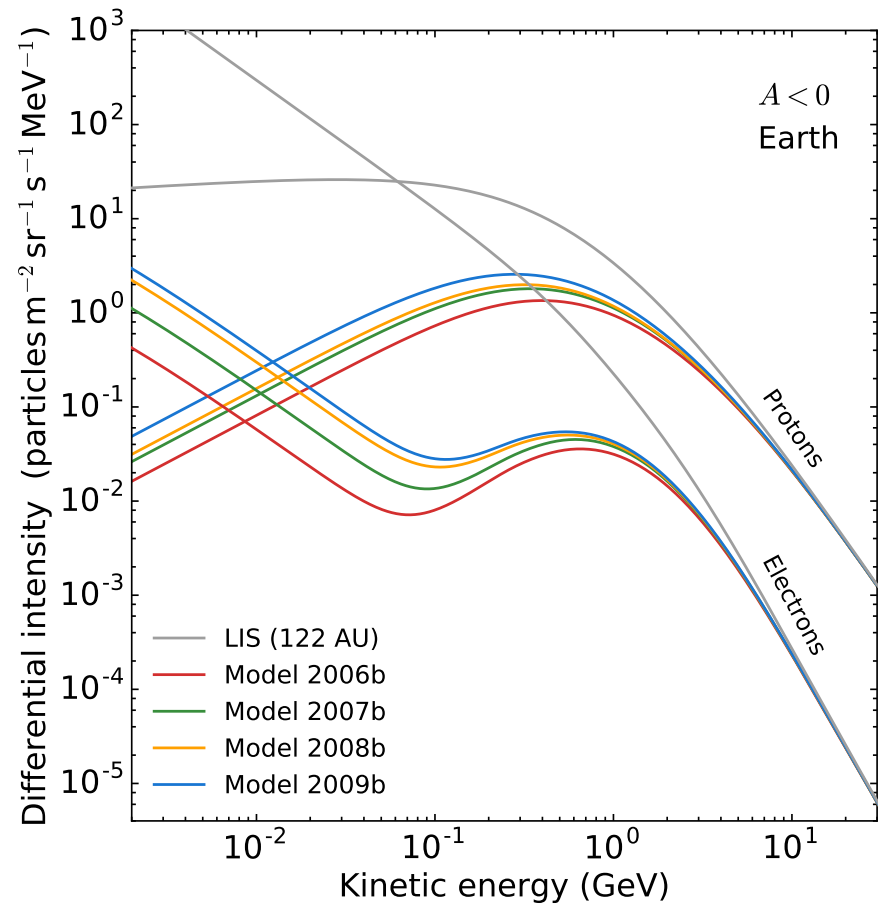

Fig. 4. Computed proton and electron spectra at the Earth for $2006 \mathrm{~b}$ (red) to $2009 \mathrm{~b}$ (blue), over an extended energy range for the $A<0$ cycle, with respect to the corresponding VLIS at $122 \mathrm{AU}$ (grey lines). We note the differences between the two VLISs and how protons and electrons are modulated differently at lower energies.

more low-energy CRs reached the Earth. To describe this effect correctly, the DCs also had to change with time, as shown in Fig. 2. Care was taken to account for Voyager 1 proton measurements in the outer heliosphere while also simultaneously reproducing the radial and latitudinal global proton gradients in the inner heliosphere between the positions of Ulysses and PAMELA as reported by Vos \& Potgieter (2016); see also De Simone et al. (2011) and Gieseler \& Heber (2016). These computed spectra are therefore considered to be representative of how galactic CR intensities changed during the 2006 to 2009 solar minimum period, and serve as a basis for studying heliospheric modulation over an extended energy range as reported below.

\section{Comparing the computed modulation of protons and electrons}

\subsection{Energy spectra}

In this section, the computed spectra for protons and electrons at the Earth for an $A<0$ HMF polarity cycle is shown over an extended energy range, from $30 \mathrm{GeV}$ down to $1 \mathrm{MeV}$, in relation to their respective VLIS. This serves as a prediction of what may have occurred at these lower energies during the special solar minimum of 2006 to 2009.

Figure 4 depicts these computed proton and electron energy spectra for the second half of every year (indicated as 2006b to $2009 \mathrm{~b}$ as before). Focussing on protons first, a unique feature of modulated proton spectra is evident in the sense that they are bent into a characteristic $E^{+1}$ slope below $\sim 100 \mathrm{MeV}$ as a result of adiabatic energy losses caused by the expanding SW. This phenomenon is most prominent deep inside the heliosphere, making it an effective and dominant modulation mechanism 
for protons at non-relativistic energies (e.g. Moraal \& Potgieter 1982; Strauss et al. 2011). Proton spectra at the Earth therefore decrease significantly more with decreasing energies than what follows from the corresponding unmodulated proton VLIS. This effect on modulated spectra gradually becomes less with increasing distance so that the modulated spectra systematically fold back into the shape of the proton VLIS at the HP. As mentioned before, over the course of the 2006 to 2009 minimum, the proton spectrum became progressively softer, with the peak of the modulated spectrum increasing from 1.35 to 2.57 particles $\mathrm{m}^{-2} \mathrm{sr}^{-1} \mathrm{~s}^{-1} \mathrm{MeV}^{-1}$, with an accompanying shift in the peak energy from $400 \mathrm{MeV}$ to $280 \mathrm{MeV}$.

Apart from being a factor of approximately 100 lower in intensity than protons above $\sim 100 \mathrm{MeV}$, modulated electron spectra shown in Fig. 4 have a notably different behaviour compared to proton spectra. In the first place, electrons experience much less adiabatic energy loss than protons, so they continue to respond to changes in the DCs even at low energies. Diffusion thus becomes the dominant modulation mechanism below $\sim 200 \mathrm{MeV}$ because the electron DCs are independent of rigidity below $\sim 200 \mathrm{MeV}$. The modulated electron spectra then take on a shape similar to that of the VLIS. As a result, modulated electron intensities begin to increase below $\sim 200 \mathrm{MeV}$, as prescribed by the increasing electron VLIS; the total amount of modulation at the Earth remains unchanged with decreasing energy, which leads to the characteristic shape of the modulated electron spectrum. Of course, changing the rigidity dependence of the DCs below this energy will directly lead to a different spectral shape, which is in contrast to how protons behave when this is done (Potgieter 1996).

Secondly, the new electron VLIS crosses the proton VLIS at $\sim 60 \mathrm{MeV}$ and becomes significantly higher toward lower the energies. The modelling over this extended energy range predicts that galactic electron intensities at the Earth should also surpass that of protons below $\sim 10 \mathrm{MeV}$. More modulation reduces the intensity so that the crossing of modulated electron and proton spectra shifts to lower energies as is shown in Fig. 4.

The contribution from Jovian electrons is not accounted for in these calculations, so that the modulated spectra shown here reflect electron intensities of galactic origin only and the spectrum for 2009 can be considered as an upper limit to how much galactic electrons may contribute to the total amount of electrons reaching the Earth at these energies. For a similar discussion, we refer also to Potgieter \& Nndanganeni (2013).

Thirdly, from 2006 to 2009, the local peak in electron intensity (located around $600 \mathrm{MeV}$ ) gradually shifts to lower energies, while the local minimum (in the region where the spectrum turns upward) shifts to higher energies, from $\sim 70 \mathrm{MeV}$ to $\sim 120 \mathrm{MeV}$. These characteristic peaks in the modulated electron spectra and the described behaviour in terms of energy occur because of the change in the interplay between drifts and diffusion, where modulation becomes more diffusion dominated for electrons towards lower energies as drifts phase out significantly, as is shown in Fig. 2.

Figure 5 gives the modulated intensity ratios of protons (top panel) and electrons (bottom panel), relative to their $2006 \mathrm{~b}$ spectrum, now shown as a function of rigidity instead of kinetic energy. For both these CR particles, the relative half-yearly increases above $\sim 10 \mathrm{GV}$ are small and become negligible above $\sim 30 \mathrm{GV}$, indicating how modulation fades out at these rigidities. The largest relative increase for protons and electrons occurred at the lowest rigidities, as expected. From 2006b to 2009b, proton intensities reached a maximum relative increase of a factor of 3.0 at $\sim 100 \mathrm{MV}$, as the result of adiabatic energy losses, which

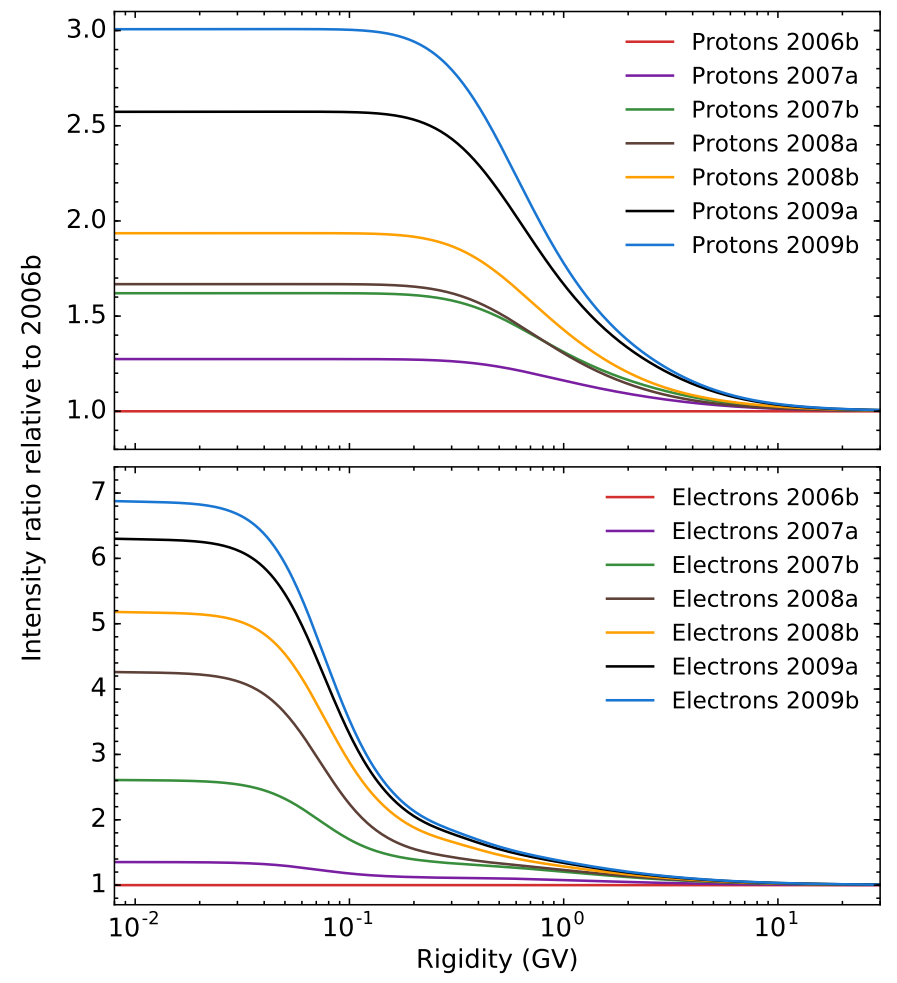

Fig. 5. Computed intensity ratios for protons (top) and electrons (bottom), relative to $2006 \mathrm{~b}$, now shown as a function of rigidity down to $10 \mathrm{MV}$. This ratio is indicative of how the amount of modulation that occurs at the Earth changes from 2006 to 2009.

become dominant at these rigidities, as discussed previously. In contrast, electron intensities continue to show larger relative increases towards lower rigidities before reaching a maximum relative increase of a factor of 6.8 at $10 \mathrm{MV}$. This once again emphasises the difference in electron and proton modulation.

The modulated $2007 \mathrm{~b}$ and $2008 \mathrm{a}$ proton spectra show very similar relative increases, which comes as a result of the sudden increase in the HCS tilt angle during the first half of 2008. Vos \& Potgieter (2015) emphasised that the net effect of this transient increase in $\alpha$ was that proton intensities were temporarily suppressed, resulting in a 2008a spectrum that is almost identical to that of $2007 \mathrm{~b}$. The HMF magnitude continued to steadily decrease throughout 2008 and the DCs consequently increased, therefore it follows that this reduction of the proton intensity is linked to HCS drifts, which are largely determined by the value $\alpha$. Since protons drift into the heliosphere mainly along the HCS, while electrons drift inwards mainly over the polar regions, the expectation that electrons should remain unaffected by this sudden increase in $\alpha$ is confirmed by what is shown for electrons in the bottom panel of Fig. 5, which is in contrast to what happens to protons over this period. Overall, the presence of drifts is supported by the modelling and PAMELA measurements; see also the discussions by Potgieter et al. (2014) and Di Felice et al. (2017).

\subsection{The effect of drifts and the HMF polarity cycle}

To further illustrate the impact of drifts on the modulation of protons and electrons, the propagation of protons and electrons is simulated for the two HMF polarity cycles. This is shown in Fig. 6 for proton spectra (top panel) and electron spectra (bottom panel) as a function of rigidity, for an $A<0$ (solid lines) 


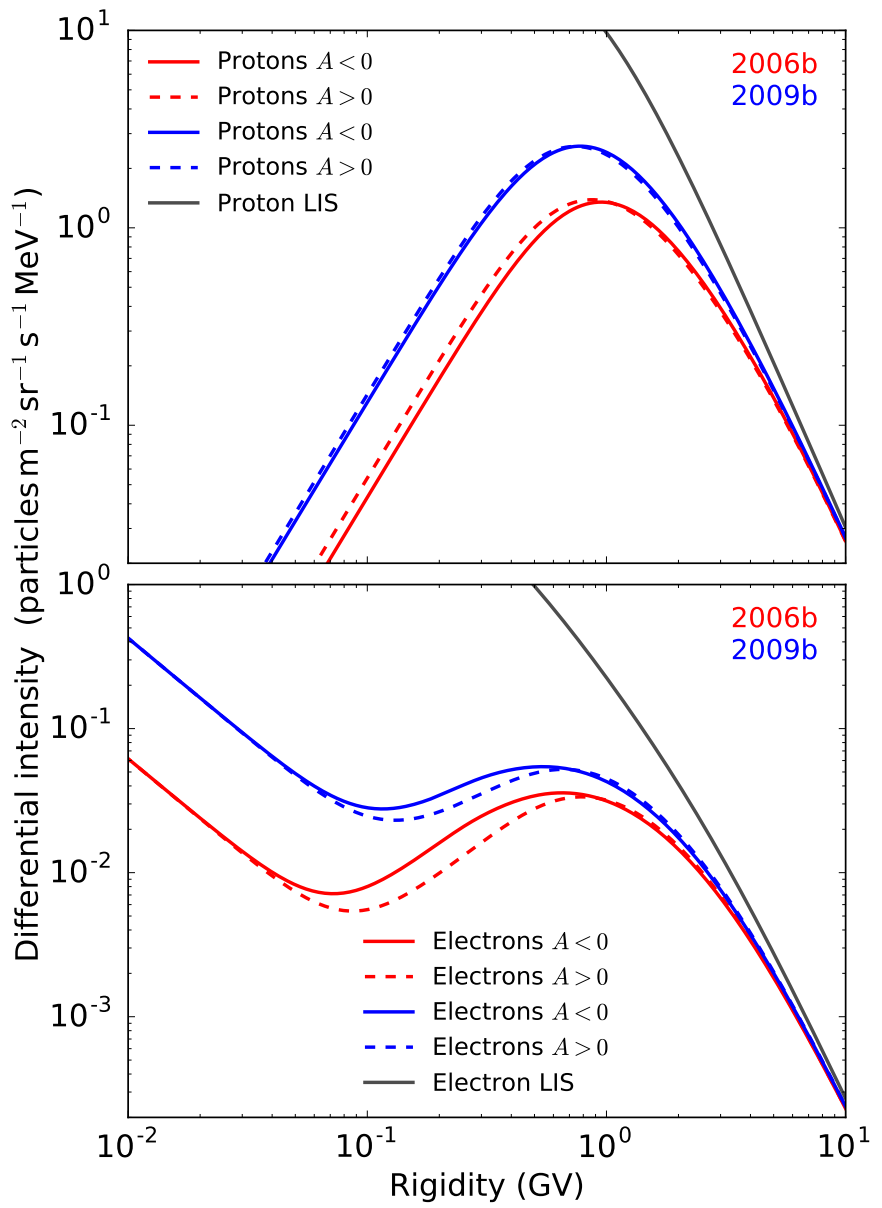

Fig. 6. Computed proton (top panel) and electron (bottom panel) rigidity spectra are shown for the $A<0$ (solid lines) and the $A>0$ (dashed lines) polarity cycles. The $2006 \mathrm{~b}$ and $2009 \mathrm{~b}$ periods are represented by the red and blue colours. Solid grey lines are the proton and electron VLIS from Fig. 1. The dashed lines are, in fact, predictions of what may be observed during the upcoming $A>0$ solar minimum period if similar conditions would prevail then as in 2006 to 2009.

and for an $A>0$ (dashed lines) polarity cycle. The red and blue colours represent spectra computed for $2006 \mathrm{~b}$ and $2009 \mathrm{~b}$, respectively. The solid lines represent spectra according to the modulation conditions from 2006 to 2009. If identical modulation conditions would occur during the next solar minimum period, with the important exception of the HMF direction, it is predicted that the proton spectrum at Earth below $\sim 1 \mathrm{GV}$ will be higher during the coming $A>0$ cycle than during the previous $A<0$ cycle; we also refer to Strauss \& Potgieter (2014b). At $\sim 1 \mathrm{GV}$, the modulated $A>0$ and $A<0$ spectra cross, so that for rigidities above $\sim 1 \mathrm{GV}$, the $A>0$ spectrum is above the $A<0$ spectrum (for more on this required crossing of consecutive solar minimum spectra, we refer to Reinecke \& Potgieter 1994). Evidently, for electrons, the predicted upcoming solar minimum spectra are lower than the equivalent spectra in 2006 and 2009, as is shown in the bottom panel of Fig. 6 .

Figure 7 shows the computed ratio of the $A>0$ to $A<0$ spectra for protons (solid lines) and electrons (dashed lines), which first quantifies the difference between spectra from opposite polarity cycles (as caused by drift effects). The largest difference between protons and electrons occurs in their behaviour at low rigidities. Below $\sim 1 \mathrm{GV}$, the $A>0$ proton spectrum remains above the $A<0$ spectrum, which is reflected by the $A>0 / A<0$

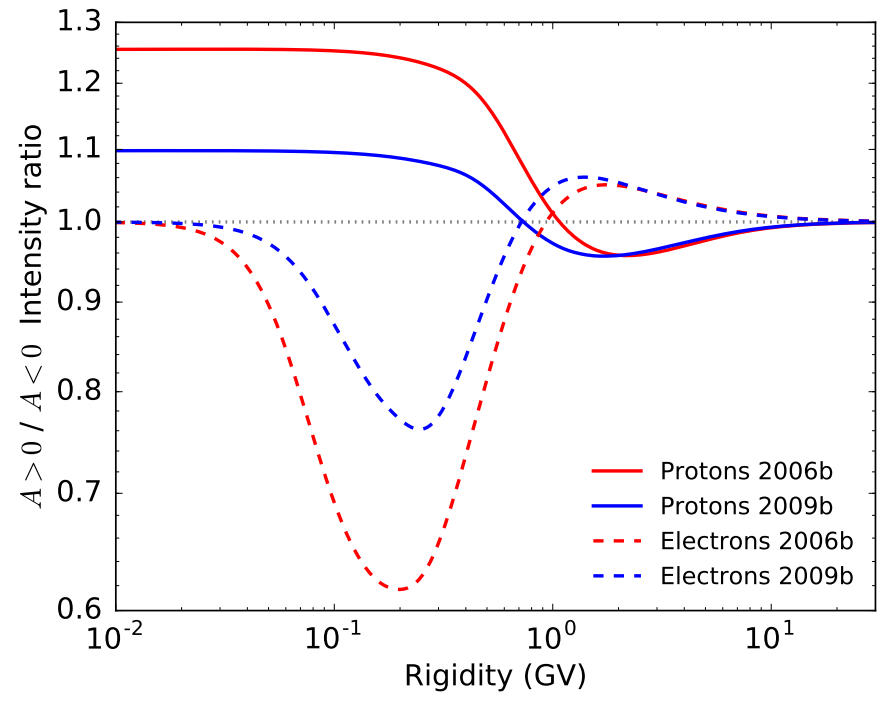

Fig. 7. Ratio of the $A>0$ to $A<0$ spectra in Fig. 6 as a function of rigidity for protons (solid lines) and electrons (dashed lines), respectively. These results serve as model predictions of how different drift effects on electrons and protons phase out with decreasing rigidity, down to rigidity values that have not been observed by PAMELA.

ratio being larger than unity, and remaining so down to the lowest rigidities. For electrons, this ratio for 2006 and 2009 decreases to its smallest values between 200 and $300 \mathrm{MV}$, where drifts are considered to have the largest effects on electrons. The ratio then increases with decreasing rigidity to reach unity eventually between 20 and $30 \mathrm{MV}$, where the $A>0$ and $A<0$ electron spectra coincide, indicating no drift effects at this rigidity. This behaviour of electrons is consistent with what was reported by Nndanganeni \& Potgieter (2016). The reason for this diverging behaviour between protons and electrons is rooted in the difference in the interplay between drifts, diffusion and adiabatic energy losses, as these particles experienced it. Diffusion begins to dominate electron modulation at low rigidities, so that no drift effects are predicted below $\sim 30 \mathrm{MV}$, resulting in the same $A>0$ and $A<0$ electron spectra where the $A>0 / A<0$ ratio becomes unity. For protons, however, drift effects are still predicted at $30 \mathrm{MV}$ and lower rigidities, as illustrated in Fig. 6.

Another noteworthy feature is the apparently smaller drift effects computed for 2009b compared to 2006b. It is explained by Potgieter et al. (2015) that the 2006 to 2009 solar minimum period appears to be relatively more diffusion-dominated than previous solar minima periods, which in turn has a significant effect on gradient, curvature and current sheet drifts. For 2006b (red lines), both protons and electrons show a larger difference between the $A>0$ and $A<0$ spectra compared to 2009b (blue lines), hence the smaller ratio of $A>0$ to $A<0$ for $2009 \mathrm{~b}$. Even though drift conditions changed from 2006 to 2009 as a result of the changes observed in $B$ (from Eq. (11)) and the tilt angle of the HCS, it is known that larger DCs (producing higher $\mathrm{CR}$ intensities) significantly reduce CR intensity gradients (less modulation) throughout the heliosphere. Because global drift effects are also determined by the value of $\nabla f$ in Eq. (4), these effects are suppressed when the DCs are increased with subsequent smaller intensity gradients; we refer also to the review by Kóta (2013). This globally contributes to the difference between the 2006b (red) and 2009b (blue) $A>0 / A<0$ ratios. Further, this subtle process of the interplay between diffusion and drift was illustrated originally by Potgieter \& Moraal (1985) and was discussed in detail by Nndanganeni \& Potgieter (2016) recently. 


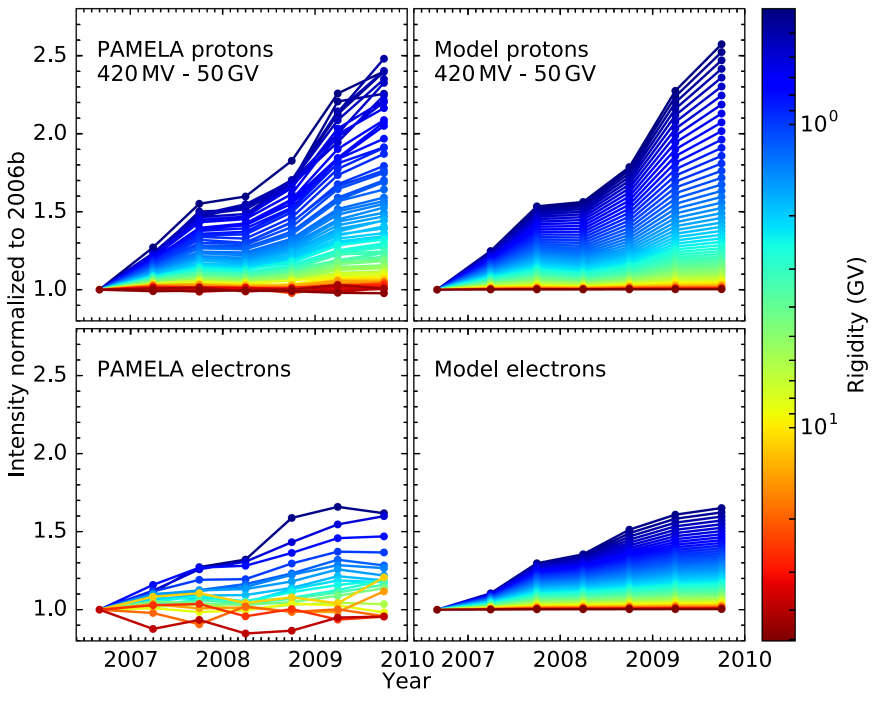

Fig. 8. Computed differential intensity increases are shown in the right panels for protons (top panel) and electrons (bottom panel) from 2006 to the end of 2009 and normalised to the intensity level of 2006b. The colour coded rigidity range is shown by the colour-bar on the right. Corresponding proton and electron intensities observed by PAMELA over the same time period are shown in the left panels.

\subsection{Unfolding of proton and electron intensities}

An alternative approach to illustrating the difference in electron and proton modulation for the period under investigation is to show how their respective fluxes for a given rigidity unfold with time. In Fig. 8, the computed increases in their differential intensity are shown in the right panels for protons (top panel) and electrons (bottom panel), normalised to the intensity level of $2006 \mathrm{~b}$. The rigidity range (420 MV to $50 \mathrm{GV}$ ) is colourcoded as shown by the colour-bar on the right. The corresponding PAMELA observations, determined in the same way as the computed values, are shown in the left panels. It is evident from both the observations and the modelled values that the proton intensities increased relatively more than the electron intensities over this period, which is reproducible with the model only when drifts are included.

These differences are further highlighted by selecting eight rigidity values over a wide range for directly comparing the behaviour of electrons to that of protons over the 2006 to 2009 period. This is shown in Fig. 9 with the rigidity values as indicated. The lower panel depicts the modelling values with the corresponding observations at $426 \mathrm{MV}$ (as the lowest available rigidity for the observed protons), $801 \mathrm{MV}, 1.60 \mathrm{GV}$ and $8.63 \mathrm{GV}$ normalised to the intensity values of 2006b. We note, firstly, the large difference between the electron and proton time profiles at $426 \mathrm{MV}$, and secondly, how this difference gradually dissipates to become almost indiscernible at $8.83 \mathrm{MV}$. Apart from being considered as evidence of charge-sign-dependent modulation, these results also illustrate how this particular modulation feature dissipates with increasing rigidity. The upper panel shows what the model predicts for lower rigidities, now with a different intensity scale than for the lower panel. For $200 \mathrm{MV}$ and $300 \mathrm{MV}$, the profiles for protons still show a somewhat steeper increase towards 2009 than the electrons, but at $100 \mathrm{MV}$ and $20 \mathrm{MV}$, the electrons increase far more than the protons. This is consistent with what was shown in Figs. 4 and 6 where it was shown how differently the electrons behave than the protons at these low rigidities, something not related to drifts. Similar results were reported by Nndanganeni \& Potgieter (2016) who modelled drift effects on electron modulation in detail.

\subsection{Electron to proton ratio}

The electron to proton intensity ratios $\left(\mathrm{e}^{-} / \mathrm{p}\right)$ are calculated next. The observed (solid lines) and modelled (dashed lines) ratios are compared in Fig. 10 as a function of time for seven rigidities, as indicated in the figure. The largest decrease in the $\mathrm{e}^{-} / \mathrm{p}$ ratio between $2006 \mathrm{~b}$ and $2009 \mathrm{~b}$ occurs at $426 \mathrm{MV}$, which is the lowest matching rigidity for PAMELA electrons and protons. At this rigidity, the ratio decreased by a factor of approximately 1.5. Consistent with Fig. 9, the $\mathrm{e}^{-} / \mathrm{p}$ ratio changes systematically less with time as the rigidity increases. PAMELA observations indicate that during the solar minimum period from 2006 to 2009 , charge-sign-dependent effects were not evident (observable) beyond 10-13 GV (Di Felice et al. 2017).

Evidently, the displayed behaviour of the $\mathrm{e}^{-} / \mathrm{p}$ ratio for rigidities between $426 \mathrm{MV}$ and $8.63 \mathrm{GV}$ is very well simulated with the modulation model (dashed lines and open circles). Predicting charge-sign-dependent modulation, Potgieter et al. (2001) showed that the largest $\mathrm{e}^{-} / \mathrm{p}$ ratio is expected during an $A<0$ cycle, which is also supported by observations made over a span of $22 \mathrm{yr}$ (e.g. Heber \& Marsden 2001; Heber \& Potgieter 2006).

Figure 11 displays the computed $\mathrm{e}^{-} / \mathrm{p}$ ratios over a wider rigidity range than what was observed by PAMELA, starting with the ratio of their respective VLIS (grey line). Below $\sim 50 \mathrm{MV}$, the $\mathrm{e}^{-} / \mathrm{p}$ ratio for both the modulated and VLISs increases above unity because the electron intensity exceeds the proton intensity at these rigidities. The opposite occurs for rigidities above $\sim 50 \mathrm{MV}$. The modulated $\mathrm{e}^{-} / \mathrm{p}$ ratio has a constant slope below $\sim 100 \mathrm{MV}$ that is steeper than the slope of the VLIS ratio because the modulated proton spectra take on the characteristic adiabatic slope at low rigidities while modulated electron spectra take on the same slope as the VLIS as shown before. The modulated ratio for the simulated $2006 \mathrm{~b}$ period is the lowest of all the modulated ratios below $\sim 100 \mathrm{MV}$, which indicates that electron intensities increased relatively more than that of protons during the consecutive half-years. However, as particle drifts come into play above $\sim 100 \mathrm{MV}$, the simulated $2006 \mathrm{~b}$ ratio becomes the highest of the modulated ratios because the proton intensities increase more than electrons over time, which is in line with previously reported drift predictions (Ferreira \& Potgieter 2004; Potgieter 2014a). From the interplay between drifts and diffusion, it is found that gradient, curvature and current sheet drifts are expected to have a maximum effect between $\sim 300 \mathrm{MV}$ and $\sim 800 \mathrm{MV}$. The factor of $\sim 1.5$ decrease in the $\mathrm{e}^{-} / \mathrm{p}$ ratio at $426 \mathrm{MV}$ can therefore be considered the maximum change in this ratio as a result of drifts, from 2006 to 2009. At higher rigidities, the decrease in the ratio becomes less steep as CRs experience less modulation and all modulation processes compete equally (see also Potgieter et al. 2014, 2015).

Finally, Fig. 12 displays the computed $\mathrm{e}^{-} / \mathrm{p}$ ratios similarly to Fig. 11 but only for the 2006 and 2009 periods, in comparison with the predicted ratios (dashed lines) for the upcoming $A>0$ solar minimum period, assuming that modulation condition will then be identical to what occurred in 2006 and 2009.

\section{Discussion and conclusions}

Particle drift in the heliosphere, as one of the four major solar modulation mechanisms, is known as the main process for causing charge-sign-dependent solar modulation. Nevertheless, the 
M. S. Potgieter and E. E. Vos: Heliospheric modulation of cosmic-ray protons and electrons
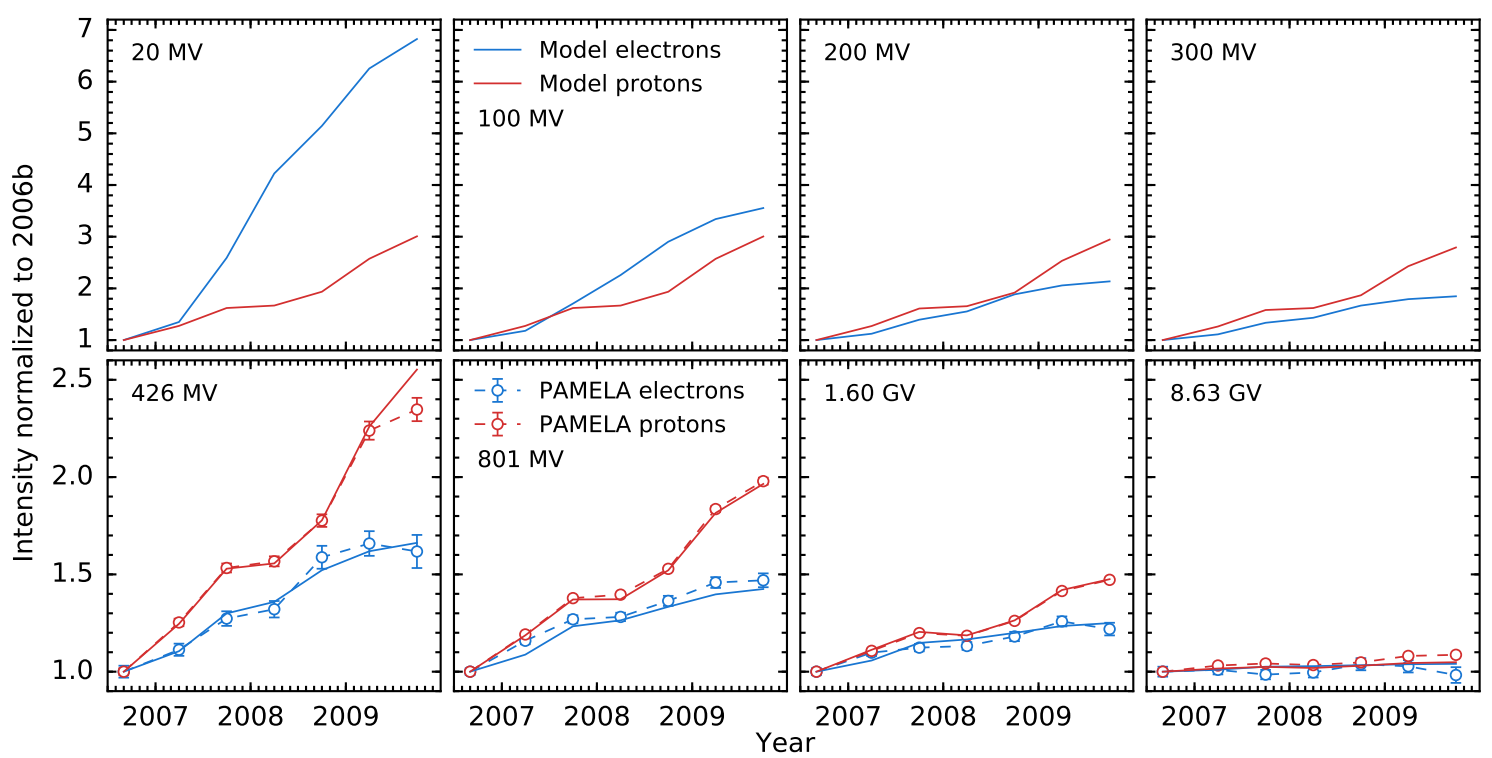

Fig. 9. A comparison of the relative intensity increases of electrons (blue lines) and protons (red lines) as a function of time for eight selected rigidities, normalised to the intensity values of 2006b. Lower panel shows a comparison of the observed values (dashed lines with open symbols) and corresponding modelled values for $426 \mathrm{MV}, 801 \mathrm{MV}, 1.60 \mathrm{GV}$ and $8.63 \mathrm{GV}$. We note how the difference dissipates with increasing rigidity. In the upper panel, we note the different intensity scale; for $200 \mathrm{MV}$ and $300 \mathrm{MV}$, the profiles for protons still show a somewhat steeper increase towards 2009 than the electrons, but at $100 \mathrm{MV}$ and $20 \mathrm{MV}$, the electrons increase far more than the protons, consistent with what was shown in Figs. 4 and 6. The response of electrons at these low rigidities is not related to particle drifts; how drift effects phase out with decreasing rigidity is shown in Fig. 7.

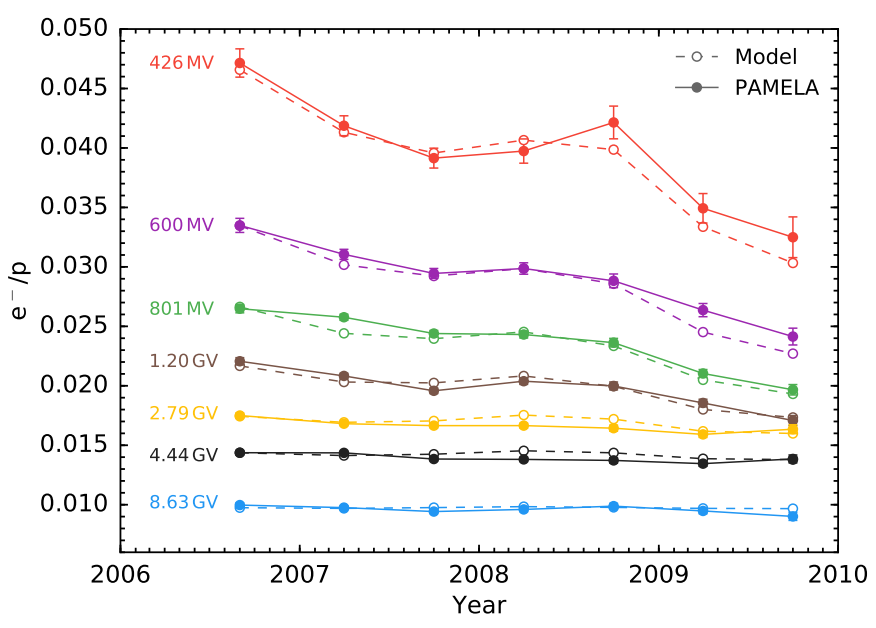

Fig. 10. Observed (solid lines) and computed (dashed lines) electron to proton ratios from 2006 to 2009 for a selection of rigidities, as indicated.

full extent of its role and its interplay with the other major mechanisms requires further investigation, as described above.

The exceptionally quiet solar minimum period from 2006 to 2009 provides optimal conditions to study drift effects during periods of very low solar activity and subsequent minimum levels of solar modulation, which may be the norm for a few more solar cycles to come; we refer to the discussion by de Jager et al. (2016). Model predictions for the next solar minimum period are shown in this context (Fig. 6).

The unprecedented accuracy of PAMELA measurements during this period (Fig. 3) has motivated this comprehensive 3D modelling of electrons and protons. First, new VLISs for electrons and protons were constructed based on Voyager 1 observations from beyond the heliopause, as shown in Fig. 1.

Simultaneously, observed galactic electron and proton spectra from PAMELA are available down to $70 \mathrm{MV}$ and $400 \mathrm{MV}$,

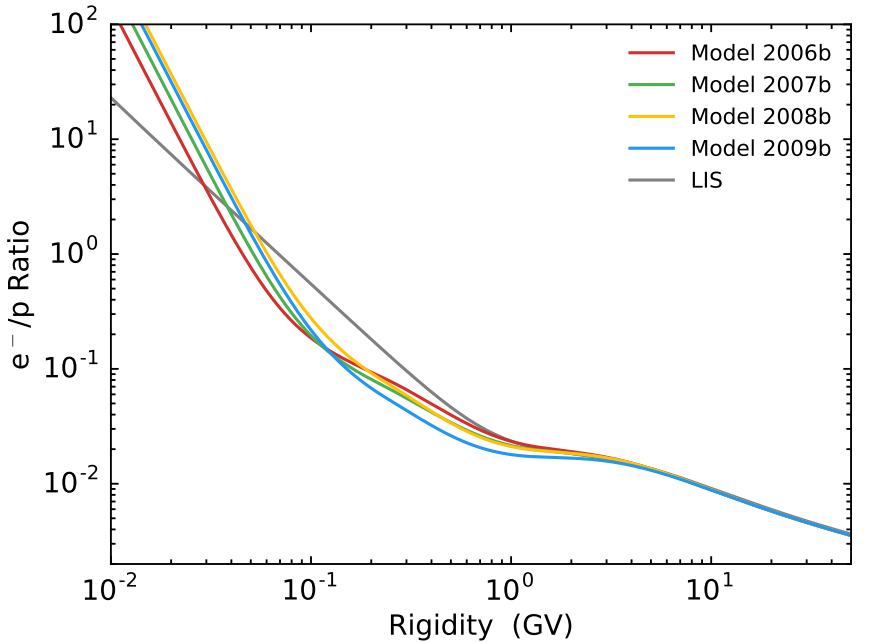

Fig. 11. Ratio of computed electron to proton intensities for their respective VLIS values (grey line) and modulated spectra at the Earth (coloured lines) over a wide rigidity range applicable to the solar minimum period from 2006 to 2009.

respectively. These observations show that as solar activity gradually decreased from 2006 to 2009, proton intensities recovered differently from electrons towards solar minimum as shown in Figs. 5 and $8-10$. These features are systematically simulated using the model to to gather insight into how drift effects played out during this exceptionally quiet period. The different ways in which drift effects dissipate for protons and electrons, particularly below $100 \mathrm{MV}$, are presented in Figs. 6 and 7. Furthermore, the rigidity dependence of the three major diffusion coefficients are presented for such quiet modulation conditions (Fig. 2). As such, it can be compared to what more elaborate diffusion theories predict. 


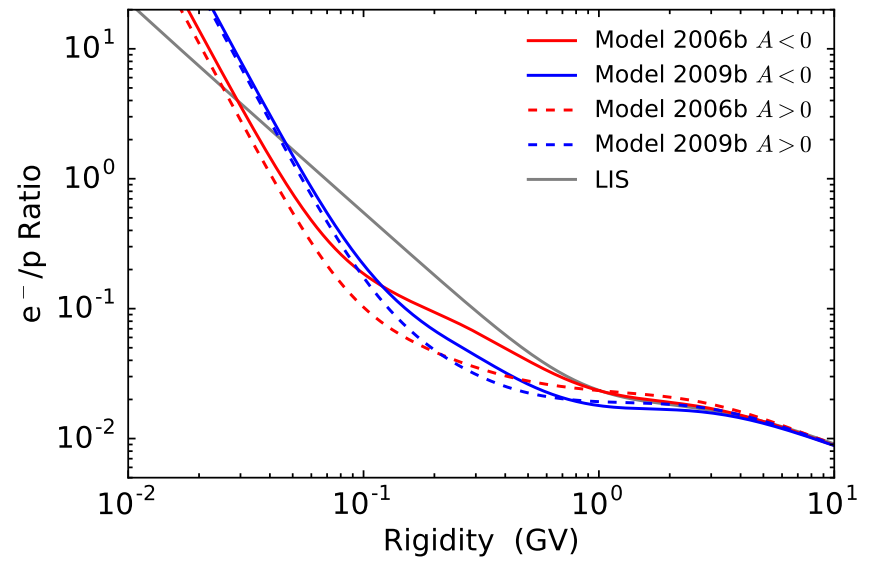

Fig. 12. Similar to Fig. 11 but now showing only the 2006 and 2009 values (solid lines) together with predicted ratios (dashed lines) for the upcoming $A>0$ solar minimum period.

Extending the model to very low rigidities, it was possible to predict $\mathrm{e}^{-} / \mathrm{p}$ ratios below $100 \mathrm{MV}$ where observations are unavailable. Predictions, shown in Figs. 6 and 7 are made for what may happen to proton and electron spectra and the corresponding $\mathrm{e}^{-} / \mathrm{p}$ ratios (Figs. 11 and 12 ) in the upcoming solar minimum, an $A>0$ period.

We conclude that the differences between proton and electron modulation observed from 2006 to 2009, and how these subside with increasing rigidity, are mostly caused by drifts, providing new and additional evidence of charge-sign-dependent solar modulation during a very quiet solar activity period. However, the model indicates that below $100 \mathrm{MV}$, drift effects gradually subside for electrons to disappear rapidly below $20 \mathrm{MV}$; the same is not seen for protons. This occurs because electron modulation becomes dominated by diffusion at lower rigidities whereas proton modulation is dominated by adiabatic cooling, which retains drift effects to the lowest rigidities (Fig. 6).

The modelling shown in Fig. 4, from $1 \mathrm{MeV}$ to $30 \mathrm{GeV}$, indicates that during solar minimum modulation, such as that observed in 2009, the modulated galactic electron intensity at the Earth starts to exceed the proton intensity around 10-20 MeV to become significantly larger the lower the energy. Based on Voyager 1 observations of electrons, the VLIS at low energies, exceed the VLIS for protons below $60 \mathrm{MeV}$. Below $10 \mathrm{MeV}$ at the Earth, galactic electrons together with Jovian electrons can be considered the dominant charged particle species.

Acknowledgements. The authors acknowledge the partial financial support of the South African National Research Foundation (NRF). They also thank Mirko Boezio, Valeria di Felice, and Riccardo Munini as members of the PAMELA team for many insightful discussions of the PAMELA data. EEV thanks SANSA, the South African Space Agency (Space Sciences), for supporting him financially during his PhD studies. He acknowledges the South African Astronomical Observatory (SAAO) and African Institute for Mathematical Sciences (AIMS) in Cape Town, South Africa, for using their facilities since September 2016.

\section{References}

Adriani, O., Barbarino, G. C., Bazilevskaya, G. A., et al. 2013, ApJ, 765, 91 Adriani, O., Barbarino, G. C., Bazilevskaya, G. A., et al. 2014, Phys. Rep., 544, 323

Adriani, O., Barbarino, G. C., Bazilevskaya, G. A., et al. 2015, ApJ, 810, 142 Aguilar, M., Alberti, G., Alpat, B., et al. 2013, Phys. Rev. Lett., 110, 1102 Aguilar, M., Aisa, D., Alpat, B., et al. 2014, Phys. Rev. Lett., 113, 1102 Aslam, O. P. M., \& Badruddin. 2012, Sol. Phys., 279, 269

Bisschoff, D., \& Potgieter, M. S. 2014, ApJ, 794, 166
Burger, R. A., Potgieter, M. S., \& Heber, B. 2000, J. Geophys. Res., 105, 27447 Chowdhury, P., Kudela, K., \& Moon, Y.-J. 2016, Sol. Phys., 291, 581 Corti, C., Bindi, V., Consolandi, C., \& Whitman, K. 2016, ApJ, 829, 8 de Jager, C., Akasofu, S.-I., Duhau, S., et al. 2016, Space Sci. Rev., 201, 109 De Simone, N., di Felice, V., Gieseler, J., et al. 2011, Astrophys. Space Sci. Trans., 7, 425

Di Felice, V., Munini, R., Vos, E. E., \& Potgieter, M. S. 2017, ApJ, 834, 89

Engelbrecht, N. E., \& Burger, R. A. 2013, ApJ, 779, 158

Engelbrecht, N. E., \& Burger, R. A. 2015, Adv. Space Res., 55, 390

Ferreira, S. E. S., \& Potgieter, M. S. 2003, Adv. Space Res., 32, 657

Ferreira, S. E. S., \& Potgieter, M. S. 2004, ApJ, 603, 744

Giacalone, J., Drake, J. F., \& Jokipii, J. R. 2012, Space Sci. Rev., 173, 283

Gieseler, J., \& Heber, B. 2016, A\&A, 589, A32

Heber, B., \& Marsden, R. G. 2001, Space Sci. Rev., 97, 309

Heber, B., \& Potgieter, M. S. 2006, Space Sci. Rev., 127, 117

Heber, B., Potgieter, M. S., \& Ferrando, P. 1997, Adv. Space Res., 19, 795

Heber, B., Wibberenz, G., Potgieter, M. S., et al. 2002, J. Geophys. Res. (Space Phys.), 107, 1274

Heber, B., Kopp, A., Gieseler, J., et al. 2009, ApJ, 699, 1956

Jokipii, J. R., \& Thomas, B. 1981, ApJ, 243, 1115

Kóta, J. 2013, Space Sci. Rev., 176, 391

Kóta, J., \& Jokipii, J. R. 1983, ApJ, 265, 573

Langner, U. W., \& Potgieter, M. S. 2004a, Adv. Space Res., 34, 144

Langner, U. W., \& Potgieter, M. S. 2004b, J. Geophys. Res., 109, A01103

Langner, U. W., Potgieter, M. S., Fichtner, H., \& Borrmann, T. 2006, ApJ, 640, 1119

le Roux, J. A., \& Potgieter, M. S. 1995, ApJ, 442, 847

le Roux, J. A., Zank, G. P., Webb, G. M., \& Khabarova, O. V. 2016, ApJ, 827, 47

Luo, X., Zhang, M., Rassoul, H. K., Pogorelov, N. V., \& Heerikhuisen, J. 2013, ApJ, 764, 85

Luo, X., Potgieter, M. S., Zhang, M., et al. 2016, ApJ, 826, 182

McComas, D. J., Ebert, R. W., Elliott, H. A., et al. 2008, Geophys. Res. Lett, 35, L18103

Menn, W., Adriani, O., Barbarino, G. C., et al. 2013, Adv. Space Res., 51, 209

Moraal, H., \& Potgieter, M. S. 1982, Astrophys. Space Sci., 84, 519

Ndiitwani, D. C., Ferreira, S. E. S., Potgieter, M. S., \& Heber, B. 2005, Ann. Geophys., 23, 61

Ngobeni, M. D., \& Potgieter, M. S. 2015, Adv. Space Res., 56, 525

Nndanganeni, R. R., \& Potgieter, M. S. 2016, Adv. Space Res., 58, 453

Parker, E. N. 1965, Planet. Space Sci., 13, 9

Picozza, P., Galper, A. M., Castellini, G., et al. 2007, Astropart. Phys., 27, 296

Potgieter, M. S. 1996, J. Geophys. Res., 101, 411

Potgieter, M. S. 2000, J. Geophys. Res., 105, 18295

Potgieter, M. S. 2013, Liv. Rev. Sol. Phys., 10, 3

Potgieter, M. S. 2014a, Adv. Space Res., 53, 415

Potgieter, M. S. 2014b, Braz. J. Phys., 44, 581

Potgieter, M. S., \& Burger, R. A. 1990, A\&A, 233, 598

Potgieter, M. S., \& Langner, U. W. 2004, ApJ, 602, 993

Potgieter, M. S., \& Moraal, H. 1985, ApJ, 294, 425

Potgieter, M. S., \& Nndanganeni, R. R. 2013, Astrophys. Space Sci., 345, 33

Potgieter, M. S., Burger, R. A., \& Ferreira, S. E. S. 2001, Space Sci. Rev., 97, 295

Potgieter, M. S., Vos, E. E., Boezio, M., et al. 2014, Sol. Phys., 289, 391

Potgieter, M. S., Vos, E. E., Munini, R., Boezio, M., \& Di Felice, V. 2015, ApJ, 810,141

Prinsloo, P. L., Potgieter, M. S., \& Strauss, R. D. 2017, ApJ, 836, 100

Raath, J. L., Potgieter, M. S., Strauss, R. D., \& Kopp, A. 2016, Adv. Space Res., 57,1965

Reinecke, J. P. L., \& Potgieter, M. S. 1994, J. Geophys. Res., 99, 14761

Shalchi, A. 2009, Nonlinear cosmic ray diffusion theories (Berlin, Germany: Springer)

Smith, C. W., \& Bieber, J. W. 1991, ApJ, 370, 435

Stone, E. C., Cummings, A. C., McDonald, F. B., et al. 2013, Science, 341, 150

Strauss, R. D., \& Potgieter, M. S. 2014a, Adv. Space Res., 53, 15

Strauss, R. D., \& Potgieter, M. S. 2014b, Sol. Phys., 289, 3197

Strauss, R. D., Potgieter, M. S., Büsching, I., \& Kopp, A. 2011, ApJ, 735, 83

Strauss, R. D., Potgieter, M. S., Büsching, I., \& Kopp, A. 2012a, Astrophys. Space Sci., 339, 223

Strauss, R. D., Potgieter, M. S., \& Ferreira, S. E. S. 2012b, Adv. Space Res., 49, 392

Strong, A. W., Orlando, E., \& Jaffe, T. R. 2011, A\&A, 534, A54

Teufel, A., \& Schlickeiser, R. 2003, A\&A, 397, 15

Vos, E. E., \& Potgieter, M. S. 2015, ApJ, 815, 119

Vos, E. E., \& Potgieter, M. S. 2016, Sol. Phys., 123, 123

Webber, W. R., \& McDonald, F. B. 2013, Geophys. Res. Lett, 40, 665

Zhao, L. L., Qin, G., Zhang, M., \& Heber, B. 2014, J. Geophys. Res. (Space Phys.), 119, 1493 